\title{
Frecuencia de Partos Pretérmino en el Hospital de la Mujer, Aguascalientes (septiembre- diciembre 2007)
}

Terrones-Saldívar MC*, Flores-Acero P**, Franco-Calderón MS**, Moreno-Anaya JJ**, Morones-García A**, Muñoz Ramírez BE**, Rosas Cabral A.**

-

\section{Resumen}

La Organización Mundial de la Salud define como par- to pretérmino (PP) aquel nacimiento que ocurre des- pués de la semana veinte y antes de la treinta y siete.

- La prematurez es responsable del 60 a $80 \%$ de las

- muertes de los recién nacidos sin malformaciones y del

- $50 \%$ de las anormalidades neurológicas de la infancia.

- El objetivo de este trabajo fue identificar la frecuen-

- cia de partos prematuros en las embarazadas que

- fueron atendidas en el Hospital de la Mujer (HM) de

- Aguascalientes, Ags, durante el I de septiembre al 31

- de diciembre del 2007. Material y Métodos: Se realizó

- un estudio observacional, descriptivo y retrospectivo. Se

- examinaron 106 expedientes de las mujeres cuyos

- partos fueron asistidos en el HM con edad gestacional

- mayor de 27 y menor de 37 semanas de gestación

- del neonato valorado por el servicio de Pediatría se-

- gún el método de Capurro. Resultados: Durante el año

- 2007 se registraron 1,075 partos pretérmino de un - total de 87 II nacimientos en el Hospital de la Mujer
(ISEA) de la ciudad de Aguascalientes, lo que representa el 12.3\%. De éstos, se revisaron 106 expedientes, el 92.5\% de ellos correspondieron a partos pretérminos entre 33 y $<37$ semanas y el resto (7.5\%) a neonatos de menos de 33 semanas; la causa principal de PP es la infección de vías urinarias (42.5\%), seguidas por la ruptura prematura de membranas (11.3\%) y la preeclampsia (9.4\%); el PP fue más frecuente en primigestas adolescentes con un adecuado control prenatal; la vía de terminación del embarazo fue la cesárea en la mayoría de los casos (71.7\%). Conclusiones: A pesar de que los neonatos pretérmino de menos de 33 semanas corresponde a un $7.5 \%$ de los casos, es en este grupo donde se espera elevada morbilidad y mortalidad neonatal. El resto de los neonatos pretérminos se adaptan adecuadamente al medio externo lo cual les permite llevarlos a alojamiento conjunto con su madre. La identificación temprana de las causas que conducen a un parto pretérmino debe de atenderse adecuadamente en primer nivel de atención médica. LUXMEDICA 2009; 4(II):19-27

Palabras clave: prematurez, Aguascalientes.

* Profesora investigadora del Centro de Ciencias Biomédicas UAA, Adscrita al Hospital de la Mujer ISEA.

** Departamento de Ginecología-Obstetricia y Pediatría, Centro de Ciencias Biomédicas UAA.

*** Profesor investigador, Centro de Ciencias Biomédicas UAA. 


\section{Introducción}

El parto pretérmino (PP) -aquel que se presenta entre las semanas 20 y 37 de gestación- es uno de los problemas perinatales de mayor prevalencia en diferentes países, incluídos los desarrollados ${ }^{1,2}$. Se presenta entre un 5 al $10 \%$ del total de nacimientos, incidencia que no ha cambiado por décadas. A pesar de los avances en la atención obstétrica, en la terapia tocolítica y el interés en la investigación de los mecanismos patogénicos, los programas para la prevención han tenido poco impacto ${ }^{3}$.

La prematurez es responsable del 60 a $80 \%$ de las muertes de los recién nacidos sin malformaciones y del $50 \%$ de las anormalidades neurológicas de la infancia. Como el riesgo de morbimortalidad en los nacimientos cercanos al término es baja, la mayor atención está focalizada en el parto pretérmino temprano (menor de 33 semanas). Si bien los nacimientos en este grupo representan el 1 al 2 $\%$ de todos los partos, ellos son responsables de cerca del $50 \%$ de la morbilidad neurológica a largo plazo y del $60 \%$ de la mortalidad neonatal ${ }^{4-7}$

El trabajo de parto prematuro espontáneo es responsable, aproximadamente, del 40 al $50 \%$ de los partos de pretérmino, mientras que el resto de estos nacimientos resultan de complicaciones obstétricas como la ruptura prematura de membranas (25 a $40 \%$ ) y de indicaciones de interrupción del embarazo antes del término (20 a $25 \%$ ) como la preclampsia ${ }^{8}$.

Se acepta que las complicaciones médicas durante la gestación, como las infecciones del tracto genital, de las vías urinarias, la anemia, la preclampsia y la ruptura prematura de membranas son los factores que se asocian al PP ${ }^{3}$. En un estudio reciente se publicó que la tasa de prematuridad es mayor en la mujeres que presentan un nivel educativo bajo, cuando se compara con las mujeres con niveles educativos altos, y que el nacimiento de niños muy prematuros (edad gestacional < 32 semanas) es dos veces más frecuente en las mujeres pertenecientes a las clases sociales mas desfavorecidas ${ }^{9}$. También varios factores conductuales aumentan el riesgo de parto de pretérmino. El hábito de fumar juega un papel bien reconocido como causa de retardo del crecimiento intrauterino más que en el parto pretérmino, sin embargo, las mujeres fumadoras tienen 20 a $30 \%$ más probabilidad de tener un parto de pretérmino ${ }^{10}$. La historia de un parto pretérmino previo sigue siendo uno de los factores de riesgo más importantes. El riesgo de recurrencia de un parto pretérmino en mujeres con antecedentes de prematuridad, oscila entre $17 \%$ y $40 \%$ y parece depender de la cantidad de partos pretérminos previos ${ }^{11}$. Un estudio reciente al estudiar el efecto a largo plazo de la prematurez encontraron una asociación negativa entre la prematurez y diversos eventos a largo plazo relacionados con la calidad de vida (superviviencia, nivel educativo y reproducción) ${ }^{12}$. 
El objetivo de este trabajo fue identificar la prevalencia de partos prematuros en mujeres atendidas en el Hospital de la Mujer $(H M)$, durante el periodo del 1 de septiembre al 31 de diciembre del 2007.

\section{| | | | | | | | | | | | | | | | | | | | | | | | | | | | | | | | | | | | | | | | | | | | | | | | | | | | | | | | | | | | | | | | | | | | | | | | | | | | | | | | | | | | | | | | | | | | | | | | | | | | | | | | | | ||}

\section{Material y método}

Se realizó un estudio descriptivo, observacional, retrospectivo y transversal. Quedaron incluídas las pacientes de cuyo parto se obtuvo un neonato calificado como pretérmino con una edad gestacional entre 27 y $<37$ semanas, de acuerdo con el método de Capurro, evaluados por el servicio de Pediatría, que fueron atendidas en el Hospital de la Mujer de Aguascalientes, entre el 1 de septiembre al 31 de diciembre del 2007. Quedaron excluídas las pacientes de partos pretérmino con feto muerto y aquellos con malformaciones fetales congénitas. Se eliminaron los expedientes incompletos. Se agruparon de acuerdo a la edad gestacional en: muy prematuros (27 a <33 SG), y prematuros (33 a <37 SG). El muestreo fue por conveniencia.
Se recolectaron los siguientes datos, edad materna, paridad, fecha de última menstruación, control prenatal (definido como adecuado con tres visitas al médico para embarazos $\leq$ a 33 semanas de gestación y 4 visitas para edad gestacional entre 33 a $<37$ semanas) presencia de complicaciones médicas durante el embarazo, resolución del embarazo, edad gestacional por Capurro, peso y Apgar del producto, destino del producto (Alojamiento Conjunto, Cunero Patológico I, (CUPA I), en esta área se hospitalizan a los niños que tienen afecciones graves, Cunero Patológico II (CUPA II), en esta área se ingresan a los neonatos para recuperar crecimiento y desarrollo y la Unidad de Cuidados Intensivos Neonatales (UCIN).

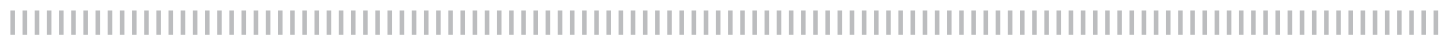

\section{Resultados}

Durante el año 2007, se registraron un total 8711 nacimientos de los cuales 1,075 partos fueron pretérmino (12.3\%) en el Hospital de la Mujer (ISEA) de la ciudad de Aguascalientes. De éstos, se revisaron 106 expedientes y se obtuvieron los siguientes datos:

De los 106 casos de PP, el 92.5\% de ellos fueron calificados con una edad gestacional entre 33 a $<37$ semanas y el $7.5 \%$ a neonatos muy prematuros (27 a $<33$ semanas) Tabla 1.

\section{Tabla I}

Distribución de casos y porcentajes de neonatos pretérmino en el Hospital de la Mujer Aguascalientes, en el período de septiembre a diciembre de 2007.

\begin{tabular}{|c|c|c|}
\hline Condición & Casos & $\%$ \\
\hline $\begin{array}{l}27 \text { a }<33 \text { SDG } \\
\text { (muy prematuros) }\end{array}$ & 8 & 7.5 \\
\hline $\begin{array}{l}33 \text { a }<37 \\
\text { (prematuros) }\end{array}$ & 98 & 92.5 \\
\hline Total & 106 & 100 \\
\hline
\end{tabular}


Las causas que condujeron al parto pretérmino fueron muy diversas, sin embargo resaltan por su mayor frecuencia la infección de vías urinarias que ocasionó 45 partos pretérmino $(42.5 \%)$; en orden decreciente le siguen la ruptura prematura de membranas (RPM) con 12 casos (11.3\%), y la pre-eclampsia con 10 (9.4\%). Cabe hacer mención que los partos pretérmino ocasionados por errores al calcular la edad gestacional por parte del clínico son 12 $(11.3 \%)$, constituyendo así otro factor que contribuye al incremento del número de partos pretérmino. Tabla 2

\section{Tabla 2}

Distribución de las causas asociadas a los partos pretérmino ocurridos en el Hospital de la Mujer durante septiembre a diciembre del 2007

\begin{tabular}{|c|c|c|}
\hline Causa & Casos & $\%$ \\
\hline Infección de vías urinarias & 45 & 42.5 \\
\hline Ruptura prematura de membranas & 12 & 11.3 \\
\hline Preeclampsia & 10 & 9.4 \\
\hline Baja reserva fetoplacentaria & 9 & 8.5 \\
\hline Diabetes mellitus descompensada & 6 & 5.7 \\
\hline Oligoamnios severo & 3 & 2.8 \\
\hline Colestasis intrahepática & 1 & 0.9 \\
\hline No especificada & 8 & 7.6 \\
\hline Error diagnóstico & 12 & 11.3 \\
\hline Total & 106 & 100 \\
\hline
\end{tabular}

La edad materna promedio fue de $24 \pm$ 6.6 años entre un rango de 12 a 39 años. La mayor frecuencia de partos pretérmino se encontró en el grupo de edad $\leq 20$ años (adolescentes); se observó que 45 (42.5\%) de los casos se encontraban en el rango de edad $\leq 20$ años; 29 (27.4\%) de las pacientes tenían entre 21 y 29 años; finalmente $32(30.2 \%)$ de ellas eran $\geq 30$ años.

\section{Tabla 3}

\section{Distribución de las pacientes que tuvieron un parto pretérmino según el grupo de edad materna}

\begin{tabular}{|l|c|c|}
\hline Edad & casos & $\%$ \\
$\leq 20$ años & 45 & 42.5 \\
\hline $21-29$ años & 29 & 27.4 \\
\hline$\geq 30$ años & 32 & 30.2 \\
\hline Total & 106 & 100 \\
\hline
\end{tabular}

De los 8 neonatos muy prematuros $(27$ a $<33$ ) pudimos observar que 4 casos tuvieron control prenatal adecuado y 4 inadecuado. Para el grupo de prematuros (98 casos) entre 33 y $<37$ semanas de ges- tación, el antecedente del control prenatal adecuado (cuatro o más visitas al médico) se documentó en el $91 \%$ de los casos, como se puede apreciar en la gráfica 1 


\section{Gráfica I}

Distribución del antecedente de control prenatal adecuado o inadecuado entre las madres de neonatos muy prematuros y prematuros

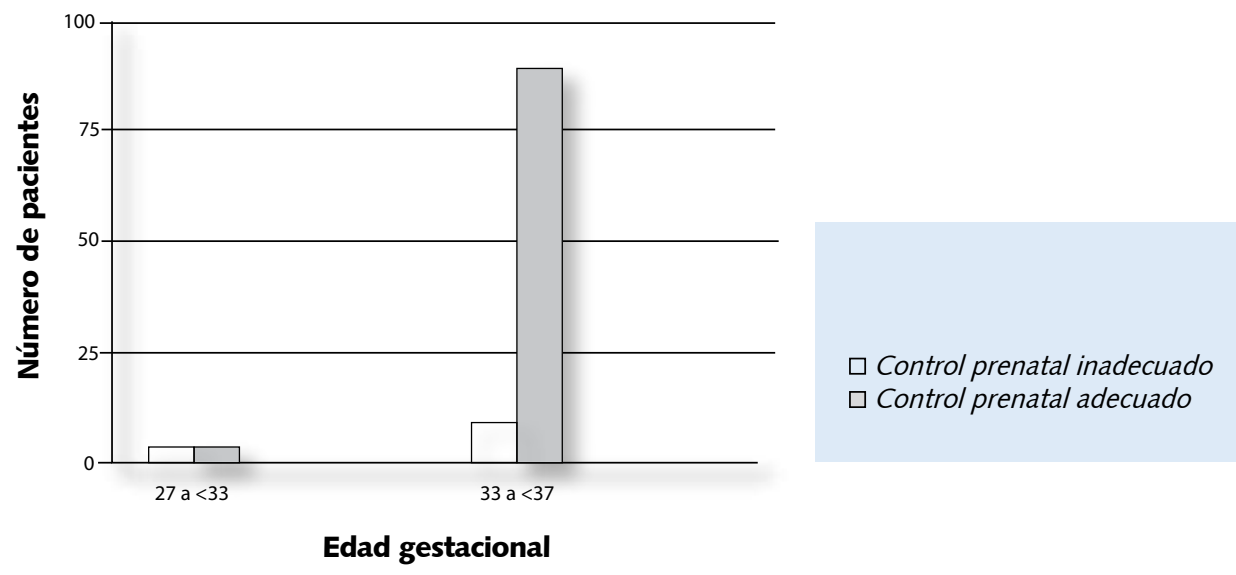

En cuanto a la paridad, casi la mitad de los partos pretérmino ocurrió en primigestas $(48.11 \%)$; de igual manera se observó

que tanto en la gesta II como en la gesta III el número de partos pretérmino, fueron 24 $(22.64 \%)$ y $23(21.70 \%)$ respectivamente.

\section{Tabla 4}

Distribución de la paridad de madres que presentaron parto pretérmino entre septiembre y diciembre del 2007 en el Hospital de la Mujer.

\begin{tabular}{|lrc|}
\hline Gesta & Casos & $\%$ \\
\hline I & 51 & 48.11 \\
\hline II & 24 & 22.64 \\
\hline III & 23 & 21.70 \\
\hline IV & 5 & 4.72 \\
\hline V & 1 & 0.94 \\
\hline VI & 1 & 0.94 \\
\hline XI & 1 & 0.94 \\
\hline TOTAL & 106 & 100.00 \\
\hline
\end{tabular}

La vía de resolución del embarazo en los partos pretérmino que se analizaron en este estudio, mostró que 76 (71.7\%) se re- solvió por vía abdominal, y 30 (28.3\%) de los casos terminaron por vía vaginal. 


\section{Gráfica 2}

Distribución de la resolución del embarazo pretérmino en el Hospital de la Mujer de septiembre a diciembre 2007

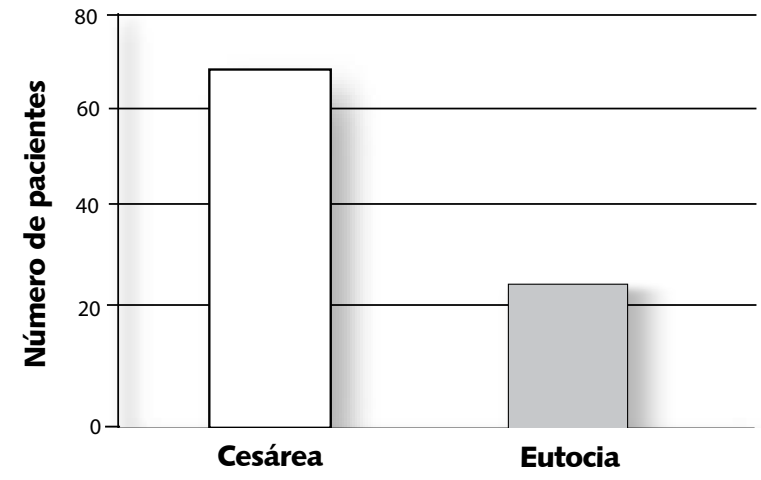

Para el grupo de muy prematuros, el de 1630 a $3600 \mathrm{~g}$ ). Vale la pena menciopromedio de peso fue de $1346 \pm 233 \mathrm{~g}$ nar que en este último grupo se registraron (rango de 970 a $1575 \mathrm{~g}$ ) y para el grupo de seis neonatos con peso mayor al esperado prematuros fue de $2494 \pm 417 \mathrm{~g}$ (rango para su edad gestacional.

\section{Gráfica 3}

Distribución del promedio de pesos entre el grupo de muy prematuros y prematuros, durante septiembre a diciembre del 2007 en el Hospital de la Mujer

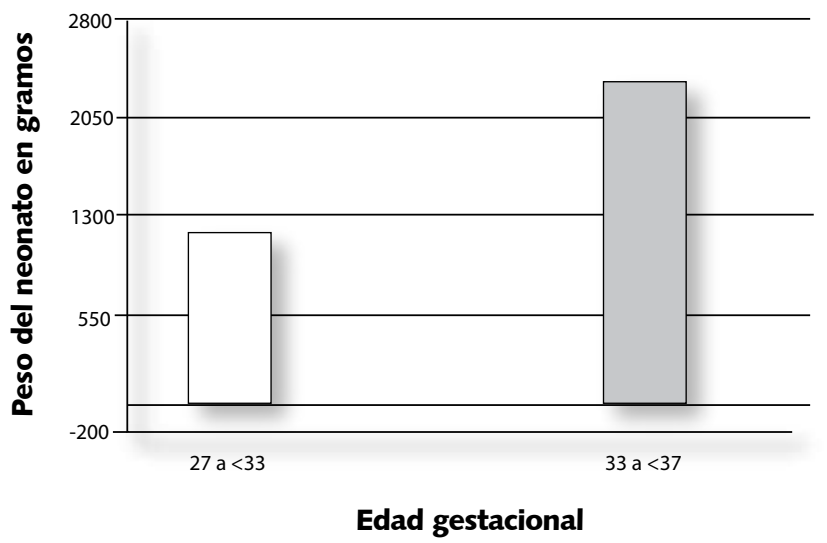

En la mayoría de los neonatos prematuros $(82 \%)$ no se requirió ningún tipo de reanimación neonatal, sólo se brindaron los cuidados inmediatos de rutina; el $14.2 \%$ requirió reanimación con presión positiva con máscara; mientras que $4(3.8 \%)$ requirió reanimación avanzada. 


\section{Tabla 5}

Tipo de reanimación requerida en productos pretérmino, durante septiembre a diciembre del 2007. Hospital de la Mujer.

\begin{tabular}{|lcc|}
\hline Tipo de reanimación & casos & $\%$ \\
\hline As & 24 & 22.6 \\
\hline AS+O2 & 63 & 59.4 \\
\hline AS+O2+PPM & 15 & 14.2 \\
\hline Reanimación avanzada & 4 & 3.8 \\
\hline TOTAL & 106 & 100.0 \\
\hline
\end{tabular}

(AS: Aspiración de Secreciones; O2: Oxígeno; PPM: Presión positiva con máscara)

La calificación de Apgar de 8-9 al minuto y cinco minutos, respectivamente, fue la puntuación más frecuente para el $57.5 \%$ de los neonatos pretérminos, que nos traduce que, en general, existe una adaptación sa- tisfactoria al medio externo, de tal manera que 62 de los casos (58.5\%) fueron enviados a alojamiento conjunto, $27(25.5 \%)$ se enviaron a CUPA I y el resto $17(16 \%)$ se enviaron a CUPA II para su tratamiento.

| | | | | | | | | | | | | | | | | | | | | | | | | | | | | | | | | | | | | | | | | | | | | | | | | | | | | | | | | | | | | | | | | | | | | | | | | | | | | | | | | | | | | | | | | | | | | | | | | | | |

\section{Discusión}

El parto pretérmino continúa siendo un desafío para la perinatología actual, en especial aquellos neonatos con edad gestacional menor a las 33 semanas porque es donde se encuentra la mayor morbilidad y la mortalidad neonatales.

En esta revisión encontramos que la prevalencia de PP en el Hospital de la Mujer es de $12.3 \%$. Comparando esta prevalencia con la reportada en países desarrollados, que oscila entre el 5 y el $11 \%$ encontramos que la nuestra es ligeramente mayor, pero menor a la reportada en algunas regiones muy pobres donde la prevalencia llega a ser hasta del $40 \%{ }^{13}$. Recientemente Osorno y cols reportaron una prevalencia del $11.9 \%$ en un hospital de concentración en Mérida Yucatán, porcentaje muy cercano al encontrado por nosotros en este estudio.

Al igual que lo reportado por otros autores, la mayoría de los neonatos corresponden a prematuros entre las 33 y $<37$ semanas de gestación (el $92.5 \%$ ) y aunque los neonatos calificados como muy prema- turos (de menos de 33 semanas) representaron el $7.5 \%$ de nuestra revisión, es en este grupo de neonatos donde se espera que hasta la quinta parte de ellos no sobrevivan al primer año y hasta el $60 \%$ de los supervivientes tendrán discapacidades neurológicas ${ }^{14}$.

El factor más frecuentemente asociado al PP fue el antecedente de la infección de vías urinarias, en el $42.5 \%$ de los casos, seguido de la RPM (11.3\%), la preclampsia $(9.4 \%)$ y el error médico en el $11.3 \%$. Estos resultados son similares a los reportados por otros autores ${ }^{3}$.

Acerca del hallazgo de que la mayoría de las pacientes que tuvieron parto pretérmino, refirieron haber acudido a sus revisiones periódicas del control prenatal, nos compromete a mejorar la calidad de la consulta obstétrica sobre todo en el primer nivel de atención, a través de acciones como la capacitación y aplicación de las guías de manejo para las causas más frecuentes de $\mathrm{PP}$, como lo es la infección de vías urinarias, ruptura prematura de membranas y preeclampsia. 
En cuanto a la edad materna, observamos que en el grupo de las adolescentes ( $<20$ años) se encuentra el mayor porcentaje de PP (42.5\%). Se acepta que en el grupo de adolescentes embarazadas se conjuntan varios factores de riesgo que ocasionan no solo PP sino también otras complicaciones obstétricas, de tal manera que nuevamente hacemos énfasis en que debemos de mejorar la calidad de la atención prenatal. Coria-Soto propone que la prematuridad puede reducirse si al menos en cada consulta de control prenatal realizamos seis procedimientos: toma de presión arterial, peso, talla, análisis de orina, de sangre y examen pélvico ${ }^{15}$.

Entre las madres estudiadas predominó la primigrávida en el $48.11 \%$ de los casos, considerando la multiparidad como el antecedente de tres o más embarazos, encontramos que una de cada tres pacientes fueron multíparas; las grandes multíparas (con siete o más embarazos) no representaron ni el $1 \%$ en este estudio.

De igual importancia es que el obstetra documente, al programar una cesárea, la edad gestacional no solo por la fecha de la última mestruación, sino también por el ultrasonido, dado que del correcto conocimiento de la edad gestacional depende en gran medida la toma de decisiones.

Por último, analizando los datos maternas de los 8 casos de neonatos muy prematuros (menores de 33 semanas por Capurro), encontramos que la mitad de los casos (4 de 8) fueron madres adolescentes multíparas, con embarazos complicados con infección de vías urinarias y RPM, con un control prenatal inadecuado en $3 / 8$, y los embarazos fueron resueltos a través de la vía abdominal en 5 de 8 casos. Es de llamar la atención que en este grupo de madres encontramos dos pacientes de 12 y 14 años de edad.

De acuerdo con nuestros resultados podemos proponer lo siguiente: 1. Es importante conocer las principales causas que conducen a partos pretérmino, en este trabajo, la infección de vías urinarias, la ruptura prematura de membranas y la preeclampsia 2. Establecer los diagnósticos de manera oportuna, de tal manera que impida el progreso hasta desembocar en un parto pretérmino. 3. Instaurar en su caso, el tratamiento adecuado.

\section{Conclusiones}

A pesar de que los neonatos pretérmino de menos de 33 semanas corresponde a un $7.5 \%$ de los casos, es en este grupo donde se espera elevada morbilidad y mortalidad neonatal. El resto de los neonatos pretérmino se adaptan adecuadamente al medio externo lo cual les permite llevarlos a alojamiento conjunto con su madre. La identificación temprana de las causas que conducen a un parto pretérmino debe de atenderse adecuadamente en primer nivel de atención médica.

Agradecemos al personal del Hospital de la Mujer por su apoyo para la realización de este trabajo, especialmente al departamento de Archivo Clínico. 


\section{Bibliografía}

1 Pallás Alonso CR, Arriaga Redondo M. Nuevos aspectos en torno a la prematuridad. Evid Pediatr, 2008;4:26

2 Swamy GK, Ostbye T, Skjaerven R. Association of preterm birth with long-term survival, reproduction, and next-generation preterm birth. JAMA 2008;299(12):1429-1436.

3 Calderón Guillén J, Vega Malagón G, Velásquez Tlapanco J, Morales Carrera R, Vega Malagón AJ. Factores de riesgo materno asociados al parto pretérmino. Rev Med IMSS 2005;43(4):339-342

4 Goeplert AL, Goldenberg RL, Andrews W. The preterm prediction studey. Am J Obstet Gynecol 2001; 184:483-488.

5 Buekens P, Wilcox A J, Kiely J, Masuy-Strooban G Birthweight, preterm birth and neonatal mortality in Belgium and the United States. Pediatr Perinat Epidemiol 1995; 2: 273-80.

6 Germain A, Carvajal J: Parto prematuro. En: Pérez A, Donoso E (eds). Obstetricia. $3^{\mathrm{a}}$ ed. Santiago, Chile: Editorial Mediterráneo, 1999.

7 Romero R, Mazor M, Muñoz H, Gómez R, Galasso $M$, Sherer DM: The preterm labor syndrome. Ann NY Acad Sci 1994; 734; 414-429.

8 Tucker JM, Goldenberg RL, Davis RO, Copper RL, Winckler $\mathrm{CL}$, Hauth JC. Etiologies of the preterm birth in an indigent population: Is prevention a logical expectation? Obstet Gynecol 1991;77:343-7

9 Thompson J, Irgens LM, Rasmussen S, Daltveit AK. Secular trends in socio-economic status and the im- plications of preterm birth. Paediatr Perinat Epidemiol. 2006;20:182-187

10 Shiono PH, Klebanoff MA, Rhoads GG. Smoking and drinking during pregnancy. JAMA 1986;255:82-84

11 Mercer BM, Goldenberg RL, Moawad AH, Meis PJ, lams JD, Das AF et al. The preterm prediction study: Effect of gestational age and cause of preterm birth on subsequent obstetric outcome. National Institute of Child Health and Human Development MaternalFetal Medicine Units Network. Am J Obstet Gynecol 1999; 181: 1216-21.

12 Perdikidis Olivieri L, Gonzalez de Dios J. Los grandes prematuros presentan menor supervivencia a largo plazo, menor nivel educativo, menor capacidad reproductiva y mayor incidencia de prematuridad en la descendencia. Evid Pediatr.2008;4:31

13 Institute of Medicine. Behrman RE, Stith BA, (eds.) Preterm birth: causes, consequences, and prevention. Washington DC: The National Academies Press, 2006.

14 Villanueva Egan LA, Contreras Gutiérrez AK, Pichardo Cuevas $M$, Rosales Lucio J. Perfil epidemiológico del parto prematuro Ginecol Obstet Mex 2008;76(9):542-548

15 Coria-Soto IL, Bobadilla JL, Notzon F. The effectiveness of antenatal care in preventing intrauterine growth retardation and low birth weight due to preterm delivery. Int Qual Health Care 1996;8:13-20 